\title{
The bus waiting area design
}

\author{
Shanghong Yu \\ Zhengzhou University of Industrial Technology, XinZheng 451100, China \\ 1069561800@qq.com
}

Keywords: Public waiting hall, man-machine, humanized, intelligent, public facilities.

\begin{abstract}
With the progress of the society and the improvement of living standards, people's demands for public facilities such as waiting hall are also increasing.For the design of the bus waiting area also requires more humane, intelligent, but the bus waiting area of our country has many shortcomings, such as the appearance of bus waiting area is relatively single, the interior design is not perfect, etc.,The existing bus waiting hall can not really meet the needs of people waiting in the life, so it is urgent to design and build a more perfect bus terminal.In this paper, the bus waiting area of the city public facilities research analysis and redesign, through the investigation and research status quo and development trend of domestic and foreign existing bus waiting areas, on everyday people when using bus waiting area has carried on the summary of existing problems, then use what they have learned in the industrial design professional knowledge, perfect design more reasonable, and more humane, intelligent bus waiting area, so that in can meet the needs of its function on the basis of, can also provide more comprehensive services for people's life.
\end{abstract}

\section{The present situation and development trend of bus waiting hall.}

\subsection{The status quo of domestic bus waiting hall.}

Bus waiting areas, generally is the bus stop matching with, is for the convenience of passengers as wind, rain, and rest, and other Settings, at present, the domestic bus waiting area basically enough on modelling beautiful, lack of innovation, and the function is not perfect enough, the existing bus waiting area can't provide good service for the public.

\subsection{Status quo of foreign bus terminals.}

Relative to the domestic bus waiting areas, foreign bus waiting areas of relatively perfect design, they will be based on what traffic to consider whether to set up a telephone booth, ATM, etc., and seats, the bin is indispensable in the basic facilities, bus waiting areas abroad besides have awnings and billboards, than domestic bus waiting area more humanized, function is more perfect, embodied in the following aspects: car stop and wait for the design of the seat; Design of special seat, telephone, city map, etc. The design of the advertisement column; The design of the waiting hall is peculiar, so it can be seen that the design of its designer is a good work of street sculpture.

\subsection{Development trend of bus terminal.}

1. Scientific. In the design of bus waiting hall, the design of bus terminal should consider the convenience of different people.

2. Artistry. The artistic quality of bus waiting hall is mainly reflected by exterior modelling, color, craft, decoration, pattern and so on. The beauty of the technical rationality of the design of the bus waiting hall; The proportion of the appropriate proportion and size of each part is beautiful.

3. Systematic. The design of the bus waiting area is different from the general product design, it is an integrated whole, and should be able to cooperate with small part of the uniform, so said bus waiting area is a systematic design, is a harmonious whole, is to address the people, the structure and function, the relationship between pattern and process.

4.Be human. As a part of public facilities, the bus waiting hall is designed to be people-oriented, to understand the physiological and psychological needs of people, and to truly reflect the respect 
and care of people.

\section{Investigation and analysis}

According to the survey, $90 \%$ of the passengers reflected the appearance and color of the existing bus terminal, which is too monotonous and uninnovative. On the basis of ergonomics knowledge, people have a relatively comfortable distance and space, fully enclosed space can give a person a kind of oppressive feeling, can't completely relax mood, so when designing bus waiting area should choose half closed or open, such ability can provide a good waiting space.

Survey found that about $80 \%$ of respondents had missed the bus stop, sit or miss the train, sometimes people are riding in a more panic, it's easy to look at the wrong stop to sit, so on the design of the bus stop we should mainly consider the feelings of the people watch stop and effect, to facilitate people to timely and accurate view. $60 \%$ of people will often don't have change, $30 \%$ of people will occasionally encounter without change, visible for most people, will often meet with the body don't have change, make people temporarily for change and might miss to ride the bus, existing bus waiting areas on this issue is not very good solution, so should be considered when designing bus waiting area how to quickly and efficiently solve the problem of change to exchange.

At the same time, for now the trash can, seat of the bus waiting area design also is not very satisfied, a lot of garbage can be placed outside the bus waiting area, cannot use in rainy day, and in the hot summer bin will send out a stink, influence people waiting; Some bus shelters do not have seats, and for those with seats that are dirty or damaged due to uncleaning, they do not provide a good resting place for people.

Therefore when designing bus waiting areas, we should not only change its shape, it should be on the part of the bus waiting area to conduct a comprehensive design, such as the height of the trash cans should accord with the requirement of human height; The design of the seat should consider not only the environment and the occasion, but also the choice of material. The location of the bus stop and billboards, color design, such as the choice of the collocation of color, material, size, all need to conduct a comprehensive consideration in the design, so as to design for satisfactory bus waiting area.

\section{3 . Design thinking}

\section{1 basic functions and analysis of bus terminal}

The design of better bus waiting hall should mainly focus on the following points:

1. Waiting function: people travel bus waiting area is to provide a temporary rest waiting space, the space need not big, but also cannot too small, in case you make people feel oppressive feeling.

2. The barrier function: bus waiting area should have a certain function of rain, so in the design of roof, we usually want to consider the height of the ceiling and covered area, the number of people can hold and keep out adopted by the way, the cover material used, transparency, etc.

3. Resting place: the chair in the bus waiting hall is simple and practical, it can not take up much space, and it should also facilitate the flow of personnel in the waiting hall.

4. Lighting system: reasonable lighting arrangement can make the passengers of the night train clearly understand the information of the train and ensure the safety of people's travel, while good lighting design can beautify the urban environment.

5. Design of station card: the bus stop sign is for passengers to provide vehicle information and route, and should have distinctive, concise and practical features.

6. Energy saving design: now the whole society advocates energy conservation, and solar energy can save resources to protect the environment and make the environment of the city more clean.

\subsection{Design of bus terminal}

Stop sign: the station card is one of the necessary equipment on the bus platform. Reasonable bus stop design can make it easier for people to find their own vehicles. When designing bus stops, it is usually necessary to consider the color and size of the font on the stop sign, the number of cars displayed on the stop sign, the direction of the car, and the night lighting of the stop sign. According 
to the ergonomic analysis, the color of the station line and the site can be white background black characters, and can also be the color with large contrast of black background yellow words, so as to facilitate passengers to view.

Bin design: a lot of trash box mouth is small, not to facilitate citizens, so when designing bus waiting areas, the height of the trash and garbage mouth, the size of the need to determine according to ergonomics and other related knowledge.

Seat: the seat design in the bus waiting hall is simple and practical, so that people can observe the vehicle information to be taken.

Change machine: for people who pay in real money, many times they will be embarrassed by the lack of change on the bus, and it will be more convenient for people to travel.

Route finder: setting up a route finder in the bus terminal can help people find the route quickly to reduce the amount of time wasted on the road.

\subsection{Project design and display}

The bus waiting hall chooses blue and white collocation, can make the whole waiting hall looks clean and neat, comfortable and natural, also do not lose contemporary feeling.

The roof of the bus waiting hall is equipped with solar panels, which can not only provide the night lighting of the waiting hall, but also save resources and protect the environment.

Buses are single direction, people waiting for the bus will be around to see when, according to the man-machine engineering design of the seat, so that makes it easier for passengers to observe the arrival of the bus information, rather than to wait and see about.

Trash barrel body discard the traditional linear and choose soft curve, make the person shine at the moment, trash can above to add a lid, can effectively prevent the rain into the trash can, such as this can give waiting passengers to provide a good waiting environment.




The change machine (left) can help passengers who find themselves without change on the bus in time to change.

The route finder (right) simply inputs your location and your destination, and can quickly offer a variety of time-saving, money-saving options.

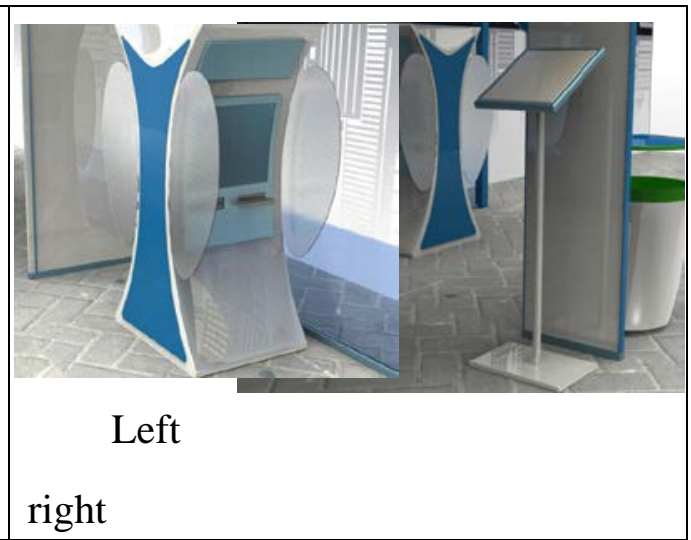

\subsection{Scheme analysis and dimension drawing}

Final design bus waiting area is modernization, from the part of the whole appearance design and interior design, such as seats, bus stop, trash, etc are based on the height of the people the most comfortable and the way to design, more humane; And the bus waiting area increases the change machine and route query the two devices, using mobile phones can't solve well get by without any change of payment problem, the problem of don't know how to ride, the whole process of waiting for the citizens of waiting more humane, intelligent, can make people's life more convenient.

After investigation and analysis, and according to the knowledge of ergonomics, the final design of the finalized case is $9540 \mathrm{~mm}$ long, $3600 \mathrm{~mm}$ wide and $2340 \mathrm{~mm}$ high.

\subsection{Material selection and processing technology}

Main frame: the main frame of the bus station waiting hall adopts the stainless steel structure, which has the features of convenient construction and assembly, strong and durable.

Organic glass ceiling: organic glass, in addition to the unparalleled high brightness, and good toughness, not easy to damage, repair, soft texture characteristics such as being strong of performance, and environmental effects of organic glass can be high recovery rate, good acid and alkali, not because of years of weathered, and produce the phenomenon such as yellowing hydrolysis, and its excellent insulation performance, easy maintenance, the rain can be their own to clean it.

Solar panel: the solar panel is made of crystalline silicon, with high efficiency of photoelectric conversion, rugged and durable, with a life span of up to 15 years and up to 25 years, saving cost.

The sign at the bus stop: general use plastic material, plastic corrosion resistance is strong, does not react with acid, alkali, low manufacture cost and easy to be with molding into different shapes, is a good insulator, edge with steel structure, surface spray treatment.

Trash can, trash can choose stainless steel material, stainless steel resistant to weak corrosive medium such as air, steam, water and acid, alkali, salt and other chemical etching medium corrosion, so the stainless steel trash can use time longer, and the bucket itself without plastic so you don't appear fade phenomenon, can guarantee and beautiful. The main processing technology is cutting, polishing and so on.

Route finder: the main body is made of stainless steel, rich in texture, screen with touch screen, easy to operate and quick to provide service to people.

Change machine: stainless steel and frosted glass. Compared to other glass partition, the ground glass partition have better safety performance, and ground glass is different from other glass partition, it is not transparent can guarantee people's privacy. The processing technology is mainly extrusion, water cutting and so on.

\section{Conclusion}

The design of this design is more unique in the design of bus waiting hall, which is not only easy to identify and modern; On the interior design to be more perfect, not only for the chairs, trash can, stop the height and size according to the man-machine engineering and so on has carried on the design again, and also increased the change machine and route query the two devices, provides the 
waiting passengers more convenience; At the same time, the waiting hall USES solar energy to supply the lighting system of the whole bus waiting hall, and the green environment beautifies the urban environment.

\section{References}

[1] zhang liang. Research on key technologies and methods of materials selection in green design [D]. Anhui: hefei university of technology,2005.

[2] zhang dongchu, pei xuming. Design of urban public facilities from industrial design [M]. Urban problems, 2003,3:21-24.

[3] wang wanting. Humanized design of bus shelter in downtown Harbin [D]. Heilongjiang: Harbin normal university,2011.

[4] liu xuetao. Research on the visual effects of city bus stations [D]. Hunan: hunan university of technology, 2011.

[5] wang anxu. Research on the development direction of urban bus stations in China [D]. Changchun: jilin art institute, 2009.

[6] guo yuan. Research on experience design of common bus waiting facilities [D]. Beijing: central academy of fine arts, 2008. 